\title{
A non-robustness in the order structure of the equilibrium set in lattice games
}

By

\author{
Andrew J. Monaco \\ Department of Economics \\ University of Kansas \\ Lawrence KS, 66045, USA \\ monacoa@ku.edu
}

\author{
Tarun Sabarwal \\ Department of Economics \\ University of Kansas \\ Lawrence KS, 66045, USA \\ sabarwal@ku.edu
}

\begin{abstract}
The order and lattice structure of the equilibrium set in games with strategic complements do not survive a minimal introduction of strategic substitutes: in a lattice game in which all-but-one players exhibit strategic complements (with one player exhibiting strict strategic complements), and the remaining player exhibits strict strategic substitutes, no two equilibria are comparable. More generally, in a lattice game, if either (1) just one player has strict strategic complements and another player has strict strategic substitutes, or (2) just one player has strict strategic substitutes and has singleton-valued best-responses, then without any restrictions on the strategic interaction among the other players, no two equilibria are comparable. In such cases, the equilibrium set is a non-empty, complete lattice, if, and only if, there is a unique equilibrium. Moreover, in such cases, with linearly ordered strategy spaces, the game has at most one symmetric equilibrium. Several examples are presented.
\end{abstract}

JEL Numbers: C70, C72

Keywords: Lattice games, strategic complements, strategic substitutes, equilibrium set

First Draft: April 2010

This Version: August 8, 2011 


\section{Introduction}

Games with strategic complements (GSC) and games with strategic substitutes (GSS) formalize two basic economic interactions and have widespread applications 1 In GSC, best-response of each player is weakly increasing (or non-decreasing) in actions of the other players, and in GSS, best-response of each player is weakly decreasing (or nonincreasing) in the actions of the other players 2

As is well-known, in GSC, the equilibrium set has nice order and structure properties: there always exist a smallest and a largest equilibrium 3 and more generally, the equilibrium set is a non-empty, complete lattice 4 These properties have proved useful in several ways; for example, they help to provide simple and intuitive algorithms to compute equilibria, and they help to show monotone comparative statics of equilibria in such games.

In contrast, in GSS, the equilibrium set is completely unordered - no two equilibria

\footnotetext{
${ }^{1}$ Such games are defined in Bulow, Geanakoplos, and Klemperer (1985), and as they show, models of strategic investment, entry deterrence, technological innovation, dumping in international trade, natural resource extraction, business portfolio selection, and others can be viewed in a more unifying framework according as the variables under consideration are strategic complements or strategic substitutes. Earlier developments are provided in Topkis (1978) and Topkis (1979).

${ }^{2}$ Versions of such games arise in diverse economic environments, including competitive strategy, public goods, industrial organization, natural resource utilization, manufacturing analysis, team management, tournaments, resource allocation, business portfolio development, principal-agent modeling, multi-lateral contracting, auctions, technological innovation, behavioral economics, and others.

${ }^{3}$ Various versions of this result can be seen in Topkis (1978), Topkis (1979), Lippman, Mamer, and McCardle (1987), Sobel (1988), Milgrom and Roberts (1990), Vives (1990), Milgrom and Shannon (1994), Echenique (2004), among others. For additional developments, confer Echenique (2002), Quah (2007), and Quah and Strulovici (2009).

${ }^{4}$ See Zhou (1994).
} 
are comparable (in the standard product order) 5 Consequently, in such games, with multiple equilibria, techniques based on the complete lattice structure of the equilibrium set, or the existence of a smallest or largest equilibrium are invalid. Typically, different techniques are required to analyze such games.

The existing results show that when we move from a setting where all players exhibit strategic complements to a setting where all players exhibit strategic substitutes, the order structure of the equilibrium set is destroyed completely. A central motivation for the present analysis is to inquire when and by how much the order structure of the equilibrium set is affected as we move player-by-player from a setting of all players with strategic complements to a setting of all players with strategic substitutes.

The new results here show that the nice order and structure properties of GSC do not survive a minimal introduction of strategic substitutes, in the following sense. Consider a lattice game 6 in which all-but-one players exhibit strategic complements (with one player exhibiting strict strategic complements 7 ), and the remaining player exhibits strict strategic substitutes 8 In this case, no two equilibria in the game are comparable (in the product order).

The results shown here are stronger, and apply to lattice games with more general strategic interaction among the players. In particular, in any lattice game, if there is reason to believe that either (1) just one player has strict strategic complements and

\footnotetext{
${ }^{5}$ See Roy and Sabarwal (2008).

${ }^{6}$ Intuitively, a lattice game is a strategic game in which every player's strategy set is a complete lattice, and every player's payoff function is continuous in own variable. No restriction is placed on strategic interaction across other players. The formal definition is given in the next section.

${ }^{7}$ Intuitively, best response is strictly increasing in other player strategies.

${ }^{8}$ Intuitively, best response is strictly decreasing in other player strategies.
} 
another player has strict strategic substitutes, or (2) just one player has strict strategic substitutes and has singleton-valued best-responses, then without any restrictions on the strategic interaction among the other players, we may conclude that no two equilibria are comparable. We present several standard examples (extended matching pennies, Cournot duopoly with spillovers, and Dove-Hawk-type game) to highlight these results 9

These results have several implications.

First, in such cases, the equilibrium set is a non-empty, complete lattice, if, and only if, the game has a unique equilibrium. In other words, with multiple equilibria, an important component (the order structure of the equilibrium set) underlying the justifiably celebrated theory of games with strategic complements does not survive a simple extension of the theory to include other realistic cases 10 Therefore, there is a need to develop new techniques to study additional cases of interest.

Second, in such cases, with multiple equilibria, techniques based on the existence of a smallest or largest equilibrium are invalid. In particular, with multiple equilibria, the standard technique of using extremal equilibria to show monotone comparative statics in GSC is invalid for the cases considered here. (In ongoing work, we show

\footnotetext{
${ }^{9}$ Games that have both strategic complements and strategic substitutes arise naturally in many applications: from the simple textbook game of matching pennies, to several examples from competitive strategy and industrial organization in Bulow, Geanakoplos, and Klemperer (1985), to games with contests in Dixit (1987), among others. Therefore, the results here have implications for a wide variety of applications.

${ }^{10}$ A quick calculation shows that in $2 \times 2$ games, with uniformly distributed payoffs, the probability of a GSC is $1 / 4$, and the probability of a game having one player with strict strategic substitutes is $3 / 4$. With more players and actions, the probability of drawing a game that falls under the cases considered here may very well be larger.
} 
that under conditions similar to those here, in parametrized lattice games, equilibria do not decrease as the parameter increases. Moreover, using new techniques, we have some preliminary results that provide conditions under which monotone comparative statics is guaranteed.)

Third, the non-ordered nature of equilibria show that starting from one equilibrium, algorithms to compute another equilibrium may be made more efficient by discarding two areas of the action space.

Fourth, if player strategy spaces are linearly ordered, then the set of symmetric equilibria is non-empty, if, and only if, there is a unique symmetric equilibrium. Therefore, in such cases, there is at most one symmetric equilibrium.

The proofs here are simple and are not meant to be a methodological contribution. Some of the simplicity arises naturally in many analyses of questions related to strategic complements and strategic substitutes. Some of it arises from the recent resurgence of work in strategic substitutes that has provided valuable insights regarding similarities and differences between GSC and GSS 11 And some of it arises from a new insight into fundamental relations underlying the order structure of the equilibrium set in the presence of strategic substitutes.

The results here are related to Roy and Sabarwal (2008), but cover cases of interest that cannot be covered in their framework. In particular, a central case in this paper, where all-but-one players have strategic complements, is ruled out by their

\footnotetext{
${ }^{11}$ For some developments in this area, confer Amir (1996), Villas-Boas (1997), Amir and Lambson (2000), Schipper (2003), Zimper (2007), Roy and Sabarwal (2008), Acemoglu and Jensen (2009), Acemoglu and Jensen (2010), Jensen (2010), Roy and Sabarwal (2010), Roy and Sabarwal (2011), among others.
} 
assumptions 12 Moreover, the proofs here are different: they are simpler and rely more directly on economic intuition.

We use the standard product order on the product of the player strategy spaces. This is a natural and intuitive order to consider in lattice games, and is used widely in GSC and in GSS. Recall that in the special case of a two-player GSS, reversing the order on the strategy space of one player transforms that game into a GSC, and results for a GSC apply to this special case. More generally, there may be no such transformation that leaves the equilibrium set invariant. For example, the textbook example of a two-player matching pennies game (a game with both complements and substitutes) has no pure-strategy Nash equilibrium, and therefore, cannot be viewed as a GSC, because a GSC always has a pure-strategy Nash equilibrium. Similarly, Roy and Sabarwal (2011) provide an example of a three-player, two-action, DoveHawk-type GSS that has no pure-strategy Nash equilibrium, and therefore, cannot be viewed as a GSC.

The paper proceeds as follows. The next section, section 2 , sets up the model, section 3 proves the main results and provides several applications, and section 4 concludes.

\footnotetext{
${ }^{12}$ This implies that the basic building blocks of the interactions studied in this paper - two-player games in which one player has strategic complements and another has strategic substitutes - are ruled out by their assumptions. In fact, none of the applications in section 3 are covered by their assumptions. Details are presented in the appendix.
} 


\section{Lattice Games}

Let $I$ be a non-empty set of players, and for each player $i$, a strategy space that is a partially ordered set, denoted $\left(X^{i}, \preceq^{i}\right)$, and a real-valued payoff function, denoted $f^{i}\left(x_{i}, x_{-i}\right)$. As usual, the domain of each $f^{i}$ is the product of the strategy spaces, $(X, \preceq)$, endowed with the product order 13 The strategic game $\Gamma=\left\{I,\left(X^{i}, \preceq^{i}, f^{i}\right)_{i \in I}\right\}$ is a lattice game if for every player $i$,

1. $X^{i}$ is a non-empty, complete lattice 14 and

2. For every $x_{-i}, f^{i}$ is order continuous in $x_{i}$

The definition of a lattice game here is very general. In particular, no restriction is placed on whether players have strategic complements or strategic substitutes. Consequently, this definition allows for general games with strategic complements, general games with strategic substitutes, and mixtures of the two.

This definition of a lattice game yields well-defined best-responses, as follows. For each player $i$, the best response of player $i$ to $x_{-i}$ is denoted $B R^{i}\left(x_{-i}\right)$, and is given by $\arg \max _{x_{i} \in X_{i}} f^{i}\left(x_{i}, x_{-i}\right)$. As the payoff function is continuous, and the strategy space is compact in the order interval topology, for every $i$, and for every $x_{-i}, B R^{i}\left(x_{-i}\right)$ is non-empty. Let $B R: X \rightarrow X$, given by $B R(x)=\left(B R^{i}\left(x_{-i}\right)\right)_{i \in I}$, denote the joint best-response correspondence. As usual, a (pure strategy) Nash equilibrium of the game is a profile of player actions $x$ such that $x \in B R(x)$.

\footnotetext{
${ }^{13}$ For notational convenience, we shall usually drop the index $i$ from the notation for the partial order.

${ }^{14}$ This paper uses standard lattice terminology. See, for example, Topkis (1998).

${ }^{15}$ In the standard order interval topology.
} 
The equilibrium set of the game is given by $\mathcal{E}=\{x \in X \mid x \in B R(x)\} 16$

Of particular interest to us are cases where the best-response of a player is either increasing (the case of strategic complements) or decreasing (the case of strategic substitutes) with respect to the strategies of the other players. Here, increasing or decreasing are with respect to an appropriately defined set order.

Recall that in a lattice game, if the payoff function of each player $i$ is quasisupermodular in $x_{i}, 17$ and satisfies the single-crossing property in $\left(x_{i} ; x_{-i}\right), 18$ then the best-response correspondence of each player is nondecreasing 19 in the standard induced set order 20 Such a game is termed a lattice game with strategic com-

\footnotetext{
${ }^{16}$ Needless to say, at this level of generality, a lattice game may have no Nash equilibrium. For example, the textbook two-player matching pennies game is admissible here, and has no pure strategy Nash equilibrium. Moreover, Roy and Sabarwal (2011) provide an example of a three-player DoveHawk-type game (a GSS) with no Nash equilibrium. One may assume additional conditions to invoke standard results to guarantee existence of equilibrium via Brouwer-Schauder type theorems, or Kakutani-Glicksberg-Ky Fan type theorems, or other types of results. We do not make these assumptions so that our results apply whenever equilibrium exists, regardless of whether a specific equilibrium existence theorem is invoked, or whether an equilibrium is shown to exist directly in a game.

${ }^{17}$ As in Milgrom and Shannon (1994), a function $f: X \rightarrow \mathbb{R}$ (where $X$ is a lattice) is quasisupermodular if (1) $f(x) \geq f(x \wedge y) \Longrightarrow f(x \vee y) \geq f(y)$, and (2) $f(x)>f(x \wedge y) \Longrightarrow f(x \vee y)>$ $f(y)$.

${ }^{18}$ A function $f: X \times T \rightarrow \mathbb{R}$ (where $X$ is a lattice and $T$ is a partially ordered set) satisfies single-crossing property in $(x ; t)$ if for every $x^{\prime} \prec x^{\prime \prime}$ and $t^{\prime} \prec t^{\prime \prime},(1) f\left(x^{\prime}, t^{\prime}\right) \leq f\left(x^{\prime \prime}, t^{\prime}\right) \Longrightarrow$ $f\left(x^{\prime}, t^{\prime \prime}\right) \leq f\left(x^{\prime \prime}, t^{\prime \prime}\right)$, and (2) $f\left(x^{\prime}, t^{\prime}\right)<f\left(x^{\prime \prime}, t^{\prime}\right) \Longrightarrow f\left(x^{\prime}, t^{\prime \prime}\right)<f\left(x^{\prime \prime}, t^{\prime \prime}\right)$.

${ }^{19} x_{-i}^{\prime} \preceq x_{-i}^{\prime \prime} \Rightarrow B R^{i}\left(x_{-i}^{\prime}\right)$ is weakly lower than $B R^{i}\left(x_{-i}^{\prime \prime}\right)$ in the induced set order. When a player's best response is a function, this translates into the standard definition of a weakly increasing function; $x_{-i}^{\prime} \preceq x_{-i}^{\prime \prime} \Rightarrow B R^{i}\left(x_{-i}^{\prime}\right) \preceq B R^{i}\left(x_{-i}^{\prime \prime}\right)$.

${ }^{20}$ The standard induced set order is defined as follows: for non-empty subsets $A, B$ of a lattice $X$, $A$ is weakly lower than $B$, if for every $a \in A$, and for every $b \in B, a \wedge b \in A$, and $a \vee b \in B$. It is
} 
plements, or GSC, for short.

In a GSC, the equilibrium set is a non-empty, complete lattice (see Zhou (1994)), and there exist a smallest equilibrium and a largest equilibrium (various versions of this result can be seen in Topkis (1978), Topkis (1979), Lippman, Mamer, and McCardle (1987), Sobel (1988), Milgrom and Roberts (1990), Vives (1990), Milgrom and Shannon (1994), among others).

Similarly, in a lattice game, if the payoff function of each player $i$ is quasisupermodular in $x_{i}$, and satisfies the decreasing single-crossing property in $\left(x_{i} ; x_{-i}\right)$,21 then the best-response correspondence of each player is nonincreasing 22 in the standard induced set order. Such a game is termed a lattice game with strategic substitutes, or GSS, for short.

Notice that the case where a player's best response is a constant function can be viewed as either strategic complements or strategic substitutes. Therefore, in a lattice game with strategic complements, strategic substitutes may be introduced trivially by having some players with constant best response functions. Of course, such games remain lattice games with strategic complements, and the equilibrium set in such games remains a non-empty, complete lattice.

sometimes also termed the strong set order.

${ }^{21}$ A function $f: X \times T \rightarrow \mathbb{R}$ (where $X$ is a lattice and $T$ is a partially ordered set) satisfies decreasing single-crossing property in $(x ; t)$ if for every $x^{\prime} \prec x^{\prime \prime}$ and $t^{\prime} \prec t^{\prime \prime},(1) f\left(x^{\prime \prime}, t^{\prime}\right) \leq$ $f\left(x^{\prime}, t^{\prime}\right) \Longrightarrow f\left(x^{\prime \prime}, t^{\prime \prime}\right) \leq f\left(x^{\prime}, t^{\prime \prime}\right)$, and $(2) f\left(x^{\prime \prime}, t^{\prime}\right)<f\left(x^{\prime}, t^{\prime}\right) \Longrightarrow f\left(x^{\prime \prime}, t^{\prime \prime}\right)<f\left(x^{\prime}, t^{\prime \prime}\right)$.

${ }^{22} x_{-i}^{\prime} \preceq x_{-i}^{\prime \prime} \Rightarrow B R^{i}\left(x_{-i}^{\prime \prime}\right)$ is weakly lower than $B R^{i}\left(x_{-i}^{\prime}\right)$ in the induced set order. When a player's best response is a function, this translates into the standard definition of a weakly decreasing function; $x_{-i}^{\prime} \preceq x_{-i}^{\prime \prime} \Rightarrow B R^{i}\left(x_{-i}^{\prime \prime}\right) \preceq B R^{i}\left(x_{-i}^{\prime}\right)$. 
For non-trivial results involving strategic substitutes, it is useful to consider players with best responses that are "strictly" decreasing with respect to the strategies of the other players. To formalize strict strategic substitutes, consider the following set order. Let $X$ be a lattice. For non-empty subsets $A, B$ of $X, A$ is strictly lower than $B$, if for every $a \in A$, and for every $b \in B, a \prec b$. This definition is a slight strengthening of the following set order defined in Shannon (1995): $A$ is completely lower than $B$, if for every $a \in A$, and for every $b \in B, a \preceq b$. Notice that when $A$ and $B$ are non-empty, complete sub-lattices of $X, A$ is strictly lower than $B$, if, and only if, $\sup A \prec \inf B$; and similarly, $A$ is completely lower than $B$, if, and only if, $\sup A \preceq \inf B$.

Strictly decreasing correspondences are defined in a natural manner using the strictly lower than set order. Let $X$ be a lattice and $T$ be a partially ordered set. A correspondence $\phi: T \rightarrow X$ is strictly decreasing, if for every $t^{\prime} \prec t^{\prime \prime}, \phi\left(t^{\prime \prime}\right)$ is strictly lower than $\phi\left(t^{\prime}\right)$. In particular, strictly decreasing correspondences that are singletonvalued are equivalent to the standard definition of a strictly decreasing function 23 Similarly, we shall also find it useful to define strictly increasing correspondences. A correspondence $\phi: T \rightarrow X$ is strictly increasing, if for every $t^{\prime} \prec t^{\prime \prime}, \phi\left(t^{\prime}\right)$ is strictly lower than $\phi\left(t^{\prime \prime}\right)$. When the correspondence is a function, this is equivalent to the standard definition of a strictly increasing function.24

\footnotetext{
${ }^{23} t^{\prime} \prec t^{\prime \prime} \Rightarrow \phi\left(t^{\prime \prime}\right) \prec \phi\left(t^{\prime}\right)$.

${ }^{24}$ Recall that Shannon (1995) provides conditions on payoff functions that guarantee a comparison in the completely lower than set order. Moreover, in finite-dimensional Euclidean spaces, Edlin and Shannon (1998) provide an additional intuitive and easy-to-use differentiable condition regarding strictly increasing marginal returns to derive a comparison in the strictly lower than set order. Both these conditions can be adapted easily for strategic substitutes.
} 
With these ideas in place, player $i$ has strict strategic substitutes, if $B R^{i}$ is strictly decreasing, and player $i$ has strict strategic complements, if $B R^{i}$ is strictly increasing.

\section{$3 \quad$ Results and Applications}

This section provides the main results in this paper and provides several applications. Theorem 1 shows that in any lattice game, one player with strict strategic substitutes and one player with strict strategic complements are sufficient to destroy the order structure of the equilibrium set, as follows.

Theorem 1. Let $\Gamma$ be a lattice game in which one player has strict strategic substitutes and another player has strict strategic complements.

If $x^{*}$ and $\hat{x}$ are distinct equilibria, then $x^{*}$ and $\hat{x}$ are not comparable.

Proof. Suppose, without loss of generality, that player 1 has strict strategic substitutes, player 2 has strict strategic complements, and suppose the distinct equilibria are comparable, with $\hat{x} \prec x^{*}$.

As case 1 , suppose $\hat{x}_{-1} \prec x_{-1}^{*}$. Then $\hat{x}_{1}=B R^{1}\left(\hat{x}_{-1}\right)$ and $x_{1}^{*}=B R^{1}\left(x_{-1}^{*}\right)$, and by strict strategic substitutes, $x_{1}^{*} \prec \hat{x}_{1}$, contradicting $\hat{x} \prec x^{*}$.

As case 2 , suppose $\hat{x}_{1} \prec x_{1}^{*}$. Then $\hat{x}_{-2} \prec x_{-2}^{*}$. Then $\hat{x}_{2} \in B R^{2}\left(\hat{x}_{-2}\right)$ and $x_{2}^{*} \in$ $B R^{2}\left(x_{-2}^{*}\right)$, and by strict strategic complements, $\hat{x}_{2} \prec x_{2}^{*}$, whence $\hat{x}_{-1} \prec x_{-1}^{*}$, and we are in case 1 . Thus $x^{*}$ and $\hat{x}$ are not comparable.

Notice the simple economic intuition in this proof. In case 1 in the proof, if opponents of player 1 play higher strategies in the $x^{*}$ equilibrium than in the $\hat{x}$ 
equilibrium, then player 1 (with strict strategic substitutes) must be playing a strictly lower strategy in the $x^{*}$ equilibrium than in the $\hat{x}$ equilibrium, and therefore, the equilibria are non-comparable. Case 2 essentially says that with $\hat{x} \prec x^{*}$, player 1 cannot be playing a higher strategy in the $x^{*}$ equilibrium. For if he did, then player 2 (with strict strategic complements) is playing a higher strategy in the $x^{*}$ equilibrium, and therefore, the opponents of player 1 are playing higher strategies in the $x^{*}$ equilibrium, whence player 1 is playing a strictly lower strategy in the $x^{*}$ equilibrium, which is a contradiction.

Notice that Roy and Sabarwal (2008) show similar results for the case where the best-response correspondence satisfies a never-increasing property. Their property is satisfied in lattice games in which every player has strict strategic substitutes, but it rules out a central case in this paper: all-but-one players have strategic complements, and the remaining player has strategic substitutes 25 In particular, none of the examples here are covered by their results. Moreover, the proofs here are different; they are simpler and rely more directly on economic intuition.

Let's look at some applications of theorem 1.

Example 1 (Matching Pennies: Double-or-Nothing). Consider the following extension of a standard matching pennies game. Each player has two pennies that they lay on a table with their hand covering the pennies. Once the pennies are revealed, the outcomes determine the payoffs as follows. Let's say that a player goes for double-or-nothing, if she plays either both heads or both tails, and she does not

\footnotetext{
${ }^{25}$ In fact, their property is not satisfied when all-but-one players have strategic complements, the remaining player has at least two actions, and there are no restrictions on the strategic interaction with the remaining player. Moreover, it is not satisfied even when these properties only hold locally. Details are provided in the appendix.
} 
go for double-or-nothing, if she plays anything else. If the outcome is $(H, H)$ and $(H, H)$, or $(T, T)$ and $(T, T)$, that is, both players go for double-or-nothing and the pennies match, then player 2 wins $\$ 2$ from player 1 . If the outcome is $(H, H) ;(T, T)$, or $(T, T) ;(H, H)$, that is, both player go for double-or-nothing and the pennies do not match, then player 1 wins $\$ 2$ from player 2 . If both players put up exactly one $H$ and one $T$, that is, nobody goes for double-or-nothing, it is a tie and no money changes hands; and if one player goes for double-or-nothing, that is, plays either $(H, H)$ or $(T, T)$, and the other does not, (that is, plays $(H, T)$ or $(T, H)$,) then the player who goes for double-or-nothing loses and pays $\$ 1$ to the other player. The payoffs of this zero-sum game are summarized in Figure 1.

Player 2

\begin{tabular}{cc|c|c|c|c|} 
& & $(\mathrm{H}, \mathrm{H})$ & $(\mathrm{T}, \mathrm{H})$ & $(\mathrm{H}, \mathrm{T})$ & \multicolumn{1}{c}{$(\mathrm{T}, \mathrm{T})$} \\
\cline { 3 - 6 } & $(\mathrm{H}, \mathrm{H})$ & $-2,2$ & $-1,1$ & $-1,1$ & $2,-2$ \\
\cline { 3 - 6 } & $(\mathrm{T}, \mathrm{H})$ & $1,-1$ & 0,0 & 0,0 & $1,-1$ \\
\cline { 3 - 6 } & $(\mathrm{H}, \mathrm{T})$ & $1,-1$ & 0,0 & 0,0 & $1,-1$ \\
\cline { 3 - 6 } & $(\mathrm{T}, \mathrm{T})$ & $2,-2$ & $-1,1$ & $-1,1$ & $-2,2$ \\
\cline { 3 - 6 } & & &
\end{tabular}

Figure 1: Matching Pennies: Double-or-Nothing

Assuming $H \prec T$, and with the standard product order, the strategy space of each player has the order $(H, H) \prec(H, T) \prec(T, T) ;(H, H) \prec(T, H) \prec(T, T)$; and $(T, H)$ and $(H, T)$ are not comparable. Notice that player 1 has strict strategic substitutes, player 2 has strict strategic complements, and the four Nash equilibria $(H, T ; T, H)$, $(H, T ; H, T),(T, H ; T, H)$, and $(T, H ; H, T)$ are all non-comparable. 
A more general version of this example is presented next.

Example 2 (A general two-player, four-point lattice game). Consider a lattice game with two players. Player 1's strategy space is a standard four-point lattice, $X_{1}=\left\{a_{1}, b_{1}, c_{1}, d_{1}\right\}$, with $b_{1}$ and $c_{1}$ unordered, and $a_{1}=b_{1} \wedge c_{1}$, and $d_{1}=b_{1} \vee c_{1}$, shown graphically in figure 2. Similarly, $X_{2}=\left\{a_{2}, b_{2}, c_{2}, d_{2}\right\}$, also shown graphically in figure 2. Suppose player 1's best response correspondence is given as follows: $B R^{1}\left(a_{2}\right)=\left\{d_{1}\right\}, B R^{1}\left(b_{2}\right)=B R^{1}\left(c_{2}\right)=\left\{b_{1}, c_{1}\right\}$, and $B R^{1}\left(d_{2}\right)=\left\{a_{1}\right\}$, and player 2 's best response correspondence is given as follows: $B R^{2}\left(a_{1}\right)=\left\{a_{2}\right\}, B R^{2}\left(b_{1}\right)=$ $B R^{2}\left(c_{1}\right)=\left\{b_{2}, c_{2}\right\}$, and $B R^{2}\left(d_{1}\right)=\left\{d_{2}\right\}$. Both are depicted in figure 2. It is easy to check that this example satisfies the conditions of theorem 1: player 1 has strict strategic substitutes, player 2 has strict strategic complements. Consequently, the four Nash equilibria $\left(b_{1}, b_{2}\right),\left(b_{1}, c_{2}\right),\left(c_{1}, b_{2}\right)$, and $\left(c_{1}, c_{2}\right)$ are all non-comparable. (Notice that double-or-nothing matching pennies is a special case of this example.)

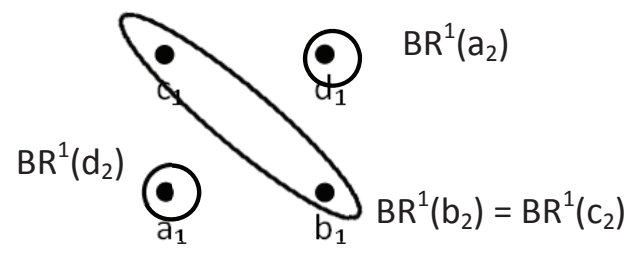

$\mathrm{X}_{1}$

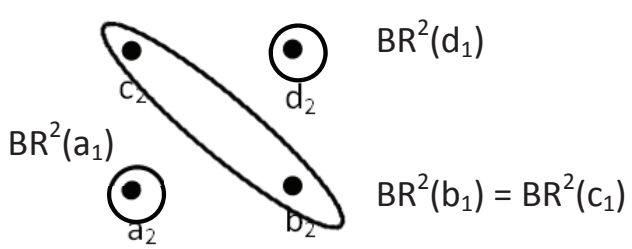

$X_{2}$

Figure 2: A General Two-Player, Four-Point Lattice Game

For completeness, observe that non-comparability of equilibria may hold with conditions weaker than one player with strict strategic complements and one player with strict strategic substitutes. The next application provides a two-player game with weak strategic substitutes (that is, best response of each player is decreasing in the completely lower than set order) and weak strategic complements (best response 
of each player is increasing in the completely lower than set order)26 that has noncomparability of equilibria.

Example 3 (Matching Pennies: Double-or-Nothing, Part 2). Consider the following modification to the game of double-or-nothing matcing pennies. If both players go for double-or-nothing and the pennies match (that is, the outcome is $(H, H)$ and $(H, H)$, or $(T, T)$ and $(T, T))$, player 2 wins $\$ 2$ from player 1 , and if both pennies do not match (the outcome is $(H, H) ;(T, T)$, or $(T, T) ;(H, H))$, player 1 wins $\$ 2$ from player 2. In all other cases, the game is a tie, and no money changes hands. The payoffs of this zero-sum game are summarized in Figure 3.

Player 2

\begin{tabular}{rr|c|c|c|c|} 
& \multicolumn{1}{c}{$(\mathrm{H}, \mathrm{H})$} & $(\mathrm{T}, \mathrm{H})$ & $(\mathrm{H}, \mathrm{T})$ & $(\mathrm{T}, \mathrm{T})$ \\
\cline { 3 - 6 } & $(\mathrm{H}, \mathrm{H})$ & $-2,2$ & 0,0 & 0,0 & $2,-2$ \\
\cline { 3 - 6 } & $(\mathrm{T}, \mathrm{H})$ & 0,0 & 0,0 & 0,0 & 0,0 \\
\cline { 3 - 6 } & $(\mathrm{H}, \mathrm{T})$ & 0,0 & 0,0 & 0,0 & 0,0 \\
\cline { 3 - 6 } & $(\mathrm{T}, \mathrm{T})$ & $2,-2$ & 0,0 & 0,0 & $-2,2$ \\
\cline { 3 - 6 } & &
\end{tabular}

Figure 3: Matching Pennies: Double-or-Nothing, Part 2

Assume the same order structure as in double-or-nothing matching pennies. Notice that player 1 has weak strategic substitutes, player 2 has weak strategic complements, and the four Nash equilibria are all non-comparable.

The intuition behind theorem 1 can be taken further, in the sense that when the best-response of the player with strict strategic substitutes is singleton-valued, the

\footnotetext{
${ }^{26}$ See the appendix for a formal definition
} 
requirement of a player with strict strategic complements can be dropped, as follows.

Theorem 2. Let $\Gamma$ be a lattice game in which one player has strict strategic substitutes, and this player's best-response is singleton-valued.

If $x^{*}$ and $\hat{x}$ are distinct equilibria, then $x^{*}$ and $\hat{x}$ are not comparable.

Proof. Suppose, without loss of generality, that player 1 has strict strategic substitutes with singleton-valued best responses, and suppose the distinct equilibria $\hat{x}$ and $x^{*}$ are comparable, with $\hat{x} \prec x^{*}$.

Case 1 remains the same as above. Suppose $\hat{x}_{-1} \prec x_{-1}^{*}$. Then $\hat{x}_{1} \in B R^{1}\left(\hat{x}_{-1}\right)$ and $x_{1}^{*} \in B R^{1}\left(x_{-1}^{*}\right)$, and by strict strategic substitutes, $x_{1}^{*} \prec \hat{x}_{1}$, contradicting $\hat{x} \prec x^{*}$.

For case 2 , suppose $\hat{x}_{-1}=x_{-1}^{*}$ and $\hat{x}_{1} \prec x_{1}^{*}$. Then $\hat{x}_{1}=B R^{1}\left(\hat{x}_{-1}\right)=B R^{2}\left(x_{-2}^{*}\right)=$ $x_{2}^{*}$, contradicting $\hat{x}_{1} \prec x_{1}^{*}$. Thus $x^{*}$ and $\hat{x}$ are not comparable.

Intuitively, in theorem 2 , if $\hat{x} \prec x^{*}$, then we need only consider the case when the opponents of player 1 play higher strategies; that is, $\hat{x}_{-1} \prec x_{-1}^{*}$. For if $\hat{x}_{-1}=x_{-1}^{*}$, then by singleton-valued best responses, the best response of player 1 to $\hat{x}_{-1}$ is the same as her best response to $x_{-1}^{*}$, and thus both equilibria are the same, which is a contradiction.

Theorem 2 formalizes the intuition that adding one player with strict strategic substitutes completely destroys the order structure of the equilibrium set. Here's an application of theorem 2 .

Example 4 (Cournot Duopoly with Spillovers). Consider two firms competing as Cournot duopolists. Let the (inverse) market demand be given by $p=a-b\left(x_{1}+x_{2}\right)$. Firm 1 has constant marginal cost, $c_{1}$; its profit is given by $\pi_{1}\left(x_{1}, x_{2}\right)=\left(a-b\left(x_{1}+\right.\right.$ $\left.\left.x_{2}\right)\right) x_{1}-c_{1} x_{1}$. Firm 1's output leads to a (perhaps) non-monotonic cost spillover 
for firm 2 , denoted $s\left(x_{1}\right)$, affecting profits of firm 2 as follows: $\pi_{2}\left(x_{1}, x_{2}\right)=(a-$ $\left.b\left(x_{1}+x_{2}\right)\right) x_{2}-c_{2} x_{2} s\left(x_{1}\right)$. Intuitively, we may view firm 1 as an established firm (or incumbent), and firm 2 as a young firm (or a relatively new entrant). The young firm receives cost externalities from the established firm, perhaps by making it easier to get industry-specific talent, or having access to superior supply chain management at a lower cost, and so on. Suppose $a=15, b=\frac{1}{2}, c_{1}=11, c_{2}=3$, and $s\left(x_{1}\right)=$ $\frac{2}{3} x_{1}^{3}-x_{1}^{2}-\frac{1}{2} x_{1}+3$. This spillover function is non-negative and non-monotonic: as firm 1's output increases from 0 to $\frac{1+\sqrt{2}}{2} \approx 1.2$, the spillover reduces from 3 to a local minimum of about 2.1, and then starts to increase.

In this case, the best responses are given by $B R_{1}\left(x_{2}\right)=\max \left\{4-\frac{1}{2} x_{2}, 0\right\}$, and $B R_{2}\left(x_{1}\right)=\max \left\{6+x_{1}+3 x_{1}^{2}-2 x_{1}^{3}, 0\right\}$. It is easy to check that there are three Nash equilibria; given by $\left(\frac{1}{2}, 7\right),(2,4)$, and $(4,0)$, and these are non-comparable, as shown in figure 4 .

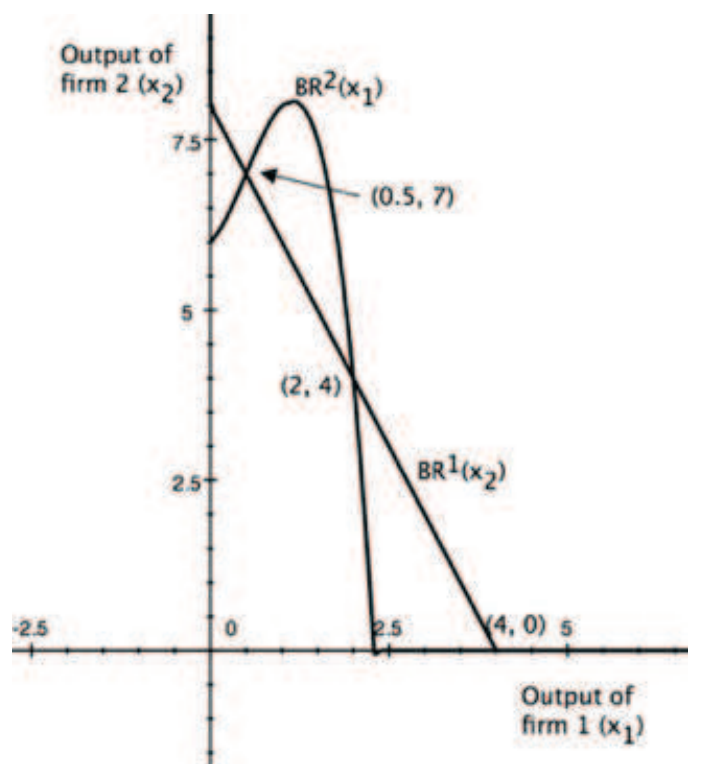

Figure 4: Cournot Duopoly with Spillovers 
Theorem 2 shows that when the best-response of the player with strict strategic substitutes is singleton-valued, the condition in theorem 1 regarding one player with strict strategic complements can be dropped. Example 5 below shows that when the best-response of the player with strict strategic substitutes is not singleton-valued, this condition in theorem 1 cannot be dropped, in general.

Example 5 (A Dove-Hawk-type game). Consider the lattice game with two players given in figure 5 , where for player $1, L \prec M \prec H$, and for player $2, L \prec$ $M$. We may interpret $L$ as a low (most Dovish, least Hawkish) action, $M$ as a medium (less Dovish, more Hawkish) action, and $H$ as a high (or least Dovish, most Hawkish) action. Player 1 has strict strategic subsitutes, with non-singleton-valued best-response: $B R^{1}(L)=\{M, H\}$, and $B R^{1}(M)=\{L\}$. Player 2 is of a type that prefers less conflict (or avoids agression, or would prefer a more "cooperative" action). Player 2 exhibits "weak" strategic complements; in fact, player 2's bestresponse function is constant, $B R^{2}(L)=B R^{2}(M)=B R^{2}(H)=\{L\}$. This game has two Nash equilibria, $(M, L)$ and $(H, L)$, and these equilibria are comparable, with $(M, L) \prec(H, L)$.

Player 2

\begin{tabular}{cc|c|c|}
\multicolumn{1}{c}{} & \multicolumn{1}{c}{$\mathrm{L}$} & $\mathrm{M}$ \\
\cline { 3 - 4 } & $\mathrm{L}$ & 0,5 & 5,0 \\
\cline { 3 - 4 } $\overrightarrow{\mathrm{D}}$ & $\mathrm{M}$ & 5,5 & 0,0 \\
\cline { 3 - 4 }$\overline{\mathrm{E}}$ & $\mathrm{H}$ & 5,5 & 0,0 \\
\cline { 3 - 4 } & &
\end{tabular}

Figure 5: A Dove-Hawk-type Game

Theorems 1 and 2 above highlight a particular non-robustness in the order struc- 
ture of the equilibrium set in lattice games.

If we consider a lattice game in which all players have strategic complements, then the equilibrium set is a non-empty, complete lattice. In particular, every pair of equilibria has a smallest larger equilibrium, and a largest smaller equilibrium.

If we modify this game to require that one player has strict strategic complements, and another has strict strategic substitutes, then we destroy the order structure of the equilibrium set completely. That is, no two equilibria are comparable.

Similarly, if we modify this game to require that one player has strict strategic substitutes, and that player's best-response is singleton-valued (perhaps because that payoff function is strictly quasi-concave), then again the order structure of the equilibrium set is destroyed completely.

Of course, the results here are stronger, and apply to general lattice games, not just to lattice games with strategic complements. In particular, in any lattice game, if there is reason to believe that either (1) one player has strict strategic complements and another player has strict strategic substitutes, or (2) just one player has strict strategic substitutes and has singleton-valued best-responses, then without any restrictions on the strategic interaction among the other players, we may conclude that no two equilibria are comparable.

Indeed, the results above yield the following corollary immediately.

Corollary 1. Let $\Gamma$ satisfy the conditions of either theorem 1 or theorem 2. The following are equivalent.

(1) $\mathcal{E}$ is a non-empty lattice

(2) $\mathcal{E}$ is a singleton

(3) $\mathcal{E}$ is a non-empty, complete lattice 
In other words, with multiple equilibria, an important component of the theory of GSC does not survive a minimal extension of the theory to include other realistic cases.

This result further implies that for the cases considered here, with multiple equilibria, techniques based on the existence of a smallest or largest equilibrium are invalid. In particular, the standard technique of using extremal equilibria to show monotone comparative statics in GSS is invalid here.

Moreover, for such cases, the non-ordered nature of equilibria show that starting from one equilibrium, algorithms to compute another equilibrium may be made more efficient by discarding two areas of the action space.

Furthermore, theorems 1 and 2 also imply immediately that when strategy spaces of players are linearly ordered 27 the game has at most one symmetric equilibrium 28 as formalized next.

Corollary 2. Let $\Gamma$ satisfy the conditions of either theorem 1 or theorem 2, and suppose the strategy space of each player is linearly ordered.

The set of symmetric equilibria is non-empty, if, and only if, there is a unique symmetric equilibrium.

\footnotetext{
${ }^{27}$ As usual, linearly ordered means that every pair of strategies is comparable. A linear order is sometimes termed a complete order.

${ }^{28} \mathrm{An}$ equilibrium is symmetric, if every player plays the same strategy in equilibrium.
} 


\section{Conclusion}

The results here show that an important component (the order structure of the equilibrium set) underlying the justifiably celebrated theory of games with strategic complements does not survive a simple extension of the theory to include other realistic cases. These results show the necessity of developing new techniques to study important questions such as monotone comparative statics; they point out a way to improve the efficiency of algorithms to compute equilibria; and they show that in fairly general cases, lattice games have at most one symmetric equilibrium.

This paper is part of an ongoing project to extend the theory of GSC to include additional realistic cases. Recent work in this area has developed new techniques that may be fruitful. Our continuing work extends these results to show that under conditions similar to those here, in parametrized lattice games, equilibria do not decrease as the parameter increases. Moreover, using new techniques, we have some preliminary results that provide conditions under which monotone comparative statics is guaranteed. 


\section{References}

Acemoglu, D., And M. K. Jensen (2009): "Aggregate comparative statics," Working Paper, Department of Economics, University of Birmingham and MIT.

(2010): "Robust comparative statics in large static games," Working Paper, Department of Economics, University of Birmingham.

Amir, R. (1996): "Cournot Oligopoly and the Theory of Supermodular Games," Games and Economic Behavior, 15, 132-148.

Amir, R., And V. E. Lambson (2000): "On the Effects of Entry in Cournot Markets," The Review of Economic Studies, 67(2), 235-254.

Bulow, J. I., J. D. Geanakoplos, and P. D. Klemperer (1985): "Multimarket Oligopoly: Strategic Substitutes and Complements," Journal of Political Economy, $93(3), 488-511$.

Dixit, A. (1987): "Strategic behavior in contests," American Economic Review, $77(5), 891-898$.

ECHENIQUe, F. (2002): "Comparative statics by adaptive dynamics and the correspondence principle," Econometrica, 70(2), 257-289.

- (2004): "A characterization of strategic complementarities," Games and Economic Behavior, 46(2), 325-347.

Edlin, A., And C. Shannon (1998): "Strict Monotonicity in Comparative Statics," Journal of Economic Theory, 81(1), 201-219.

Jensen, M. K. (2010): "Aggregative Games and Best-Reply Potentials," Economic Theory, 43(1), 45-66.

Lippman, S. A., J. W. Mamer, and K. F. MCCardle (1987): "Comparative Statics in non-cooperative games via transfinitely iterated play," Journal of Economic Theory, 41(2), 288-303.

Milgrom, P., and J. Roberts (1990): "Rationalizability, learning, and equilibrium in games with strategic complementarities," Econometrica, 58(6), 1255-1277.

Milgrom, P., and C. Shannon (1994): "Monotone Comparative Statics," Econometrica, 62(1), 157-180.

QuAh, J. K.-H. (2007): "The Comparative Statics of Constrained Optimization Problems," Econometrica, 75(2), 401-431. 
Quah, J. K.-H., And B. Strulovici (2009): "Comparative statics, informativeness, and the interval dominance order," Econometrica, 77(6), 1949-1992.

Roy, S., and T. Sabarwal (2008): "On the (Non-)Lattice Structure of the Equilibrium Set in Games With Strategic Substitutes," Economic Theory, 37(1), 161-169.

- (2010): "Monotone comparative statics for games with strategic substitutes," Journal of Mathematical Economics, 46(5), 793-806.

(2011): "Characterizing stability properties in games with strategic substitutes," Working paper series, number 201003, University of Kansas.

SCHIPPER, B. C. (2003): "Submodularity and the evolution of Walrasian behavior," International Journal of Game Theory, 32, 471-477.

Shannon, C. (1995): "Weak and Strong Monotone Comparative Statics," Economic Theory, 5(2), 209-227.

Sobel, J. (1988): "Isotone comparative statics in supermodular games," Mimeo. SUNY at Stony Brook.

Topkis, D. (1978): "Minimizing a submodular function on a lattice," Operations Research, 26, 305-321.

— (1979): "Equilibrium points in nonzero-sum n-person submodular games," SIAM Journal on Control and Optimization, 17(6), 773-787.

— (1998): Supermodularity and Complementarity. Princeton University Press.

Villas-Boas, J. M. (1997): "Comparative Statics of Fixed Points," Journal of Economic Theory, 73(1), 183-198.

Vives, X. (1990): "Nash Equilibrium with Strategic Complementarities," Journal of Mathematical Economics, 19(3), 305-321.

Zhou, L. (1994): "The Set of Nash Equilibria of a Supermodular Game is a Complete Lattice," Games and Economic Behavior, 7(2), 295-300.

ZIMPER, A. (2007): "A fixed point characterization of the dominance-solvability of lattice games with strategic substitutes," International Journal of Game Theory, 36(1), 107-117. 


\section{Appendix}

Roy and Sabarwal (2008) assume that the best-response correspondence satisfies a never-increasing property, defined as follows. Let $X$ be a lattice and $T$ be a partially ordered set. A correspondence $\phi: T \rightarrow X$ is never increasing, if for every $t^{\prime} \prec t^{\prime \prime}$, for every $x^{\prime} \in \phi\left(t^{\prime}\right)$, and for every $x^{\prime \prime} \in \phi\left(t^{\prime \prime}\right), x^{\prime} \npreceq x^{\prime \prime} 29$ This property is satisfied in lattice games in which every player has strict strategic substitutes, but it rules out a central case in this paper: all-but-one players have strategic complements, and the remaining player has strategic substitutes. To see this, consider the following.

Let $X$ be a lattice and $T$ be a partially ordered set. A correspondence $\phi: T \rightarrow X$ is weakly completely increasing, if for every $t^{\prime} \prec t^{\prime \prime}, \phi\left(t^{\prime}\right)$ is completely lower than $\phi\left(t^{\prime \prime}\right) 30$ Player $i$ has weak strategic complements, if $B R^{i}$ is weakly completely increasing 31 We have the following proposition.

Proposition 1. Let $\Gamma$ be a lattice game in which all-but-one players exhibit weak strategic complements, and the remaining player has at least two actions. The best response correspondence in such a game does not satisfy the never-increasing property.

Proof. Suppose, without loss of generality, that all-but-player-1 have weak strategic complements. Consider $x_{1}^{\prime} \prec x_{1}^{\prime \prime}$ in $X_{1}$, and $x_{-1}^{\prime} \in X_{-1}$. Then $\left(x_{1}^{\prime}, x_{-1}^{\prime}\right) \prec\left(x_{1}^{\prime \prime}, x_{-1}^{\prime}\right)$. Let $y_{1}^{\prime} \in B R^{1}\left(x_{-1}^{\prime}\right)$. For each $i \neq 1$, let $x_{-i}^{\prime}=\left(x_{1}^{\prime}, x_{-(1, i)}^{\prime}\right)$ and $x_{-i}^{\prime \prime}=\left(x_{1}^{\prime \prime}, x_{-(1, i)}^{\prime}\right)$. Then for each $i \neq 1, x_{-i}^{\prime} \prec x_{-i}^{\prime \prime}$. For each such $i$, fix $y_{i}^{\prime} \in B R^{i}\left(x_{-i}^{\prime}\right)$ and $y_{i}^{\prime \prime} \in$ $B R^{i}\left(x_{-i}^{\prime \prime}\right)$ arbitrarily. By weak strategic complements, $y_{i}^{\prime} \preceq y_{i}^{\prime \prime}$. Thus, $\left(x_{1}^{\prime}, x_{-1}^{\prime}\right) \prec$

\footnotetext{
${ }^{29}$ When best-responses are functions, this coincides with the definition of a not-increasing function, $t^{\prime} \prec t^{\prime \prime} \Rightarrow \phi\left(t^{\prime}\right) \npreceq \phi\left(t^{\prime \prime}\right)$, and in linearly ordered $X$, this is equivalent to a strictly decreasing function.

${ }^{30}$ When the correspondence is a function, this is equivalent to the standard definition of a weakly increasing function.

${ }^{31}$ Notice that strict strategic complements implies weak strategic complements.
} 
$\left(x_{1}^{\prime \prime}, x_{-1}^{\prime}\right),\left(y_{1}^{\prime}, y_{-1}^{\prime}\right) \in B R\left(x_{1}^{\prime}, x_{-1}^{\prime}\right),\left(y_{1}^{\prime}, y_{-1}^{\prime \prime}\right) \in B R\left(x_{1}^{\prime \prime}, x_{-1}^{\prime}\right)$, and $\left(y_{1}^{\prime}, y_{-1}^{\prime}\right) \preceq\left(y_{1}^{\prime}, y_{-1}^{\prime \prime}\right)$, contradicting the never-increasing property.

Consequently, the case where all-but-one players exhibit weak strategic complements, and the remaining player has strategic substitutes is not covered by Roy and Sabarwal (2008). It is precisely this case that starts the analysis in this paper: consider a movement away from the case of all strategic complements by introducing one player with strategic substitutes.

It is easy to see that the global nature of the definition of a never-increasing correspondence rules out more cases. In particular, a similar proof shows that cases with local weak strategic complements for the remaining players do not satisfy the never-increasing property. The Cournot duopoly with spillovers provides an intuitive example. In this case, player 2 does not have weak strategic complements. Nevertheless, the best-response correspondence does not satisfy the never-increasing property, because for example, for all $\epsilon>0$ sufficiently small, $\left(\frac{1}{2}, 7\right) \prec\left(\frac{1}{2}+\epsilon, 7\right)$, but $B R^{2}\left(\frac{1}{2}\right) \prec B R^{2}\left(\frac{1}{2}+\epsilon\right)$, and therefore, $B R\left(\frac{1}{2}, 7\right) \prec B R\left(\frac{1}{2}+\epsilon, 7\right)$. This occurs, because player 2 has weak strategic complements in a neighborhood of $\frac{1}{2}$, even though he does not have weak strategic complements globally. 